\title{
Philosophiques
}

\section{Hébert, Robert, Mobiles du discours philosophique : recherche sur le concept de réflexion. Montréal, Hurtubise HMH, collection Brèches, 1978, 191 pages.}

\section{Jean-Paul Brodeur}

Volume 6, numéro 1, avril 1979

URI : https://id.erudit.org/iderudit/203111ar

DOI : https://doi.org/10.7202/203111ar

Aller au sommaire du numéro

Éditeur(s)

Société de philosophie du Québec

ISSN

0316-2923 (imprimé)

1492-1391 (numérique)

Découvrir la revue

Citer cet article

Brodeur, J.-P. (1979). Hébert, Robert, Mobiles du discours philosophique :

recherche sur le concept de réflexion. Montréal, Hurtubise HMH, collection

Brèches, 1978, 191 pages. Philosophiques, 6(1), 131-145.

https://doi.org/10.7202/203111ar d'utilisation que vous pouvez consulter en ligne.

https://apropos.erudit.org/fr/usagers/politique-dutilisation/ 
HÉBERT, Robert, Mobiles du discours philosophique : recherche sur le concept de réflexion. Montréal, Hurtubise $\mathrm{HMH}$, collection Brèches, 1978. 191 pages.

par Jean-Paul Brodeur

0. Dans sa préface au Tractatus, Wittgenstein écrit : «Ce livre ne sera peut-être compris que par quelqu'un qui a déjà lui-même entretenu une fois les pensées qui y sont exprimées - ou encore des pensées similaires. "Cette remarque de Wittgenstein peut s'appliquer, toutes proportions gardées, au livre que Robert Hébert vient de faire paraitre chez Hurtubise HMH, dans la collection Brèches, dirigée par Georges Leroux.

L'un des multiples paradoxes dont joue ce livre est de pratiquer sur la dérive philosophique du concept de réflexion une sémantique sauvage (par. 9, p. 18) ${ }^{1}$ dont les procédures, ainsi que les résultats, ne peuvent être entrevus qu'au prix d'une dépense immodeste de culture. (Qui percevra, par exemple, que sous le terme francisé de " proxémique »- par. 67, p. 82 -, Robert Hébert se réfere à cette pointe ésotérique de la sémiotique, qui se pratique sous le nom de «Proxemics» dans le monde intellectuel anglo-saxon ?). L'apparition imprévue de la tripartition des fonctions articulant le monde indo-européen, d'après les travaux de Dumézil, à la fin de Mobiles $d u$ discours philosophique, nous démontre d'une autre façon encore que quelle que soit la sauvagerie de la pratique de Robert Hébert, elle n'a pas renoncé à utiliser des machines théoriques qu'on n'a pas accoutumé d'associer avec l'ingénuité d'une technologie primitive.

Je veux signifier par cette caustique entrée en matière que je ne suis pas insensible à l'embarras et/ou à l'irritation que peut susciter le caractère amphigourique de certaines des formules préméditées par Robert Hébert et, de façon particulière, des énoncés qui sont imprimés en italiques: par

1. Le livre de Robert Hébert est divisé en paragraphes (groupes de paragraphes) numérotés. En reportant le lecteur à des passages de ce livre, je citerai à la fois le numéro du paragraphe auquel je me réfere et celui de la page à laquelle je renvoie. 
exemple, «Le penser est cet agent de métaphorisation métaphorisé qui initie sémantiquement ce de quoi il se différentie idéologiquement" (par. 15, p. 26, souligné dans le texte). L'apparition parfois abrupte de telles formulations, dont la terminologie, assez vague, n'est soumise à aucune réglementation repérable, nous laisse davantage déconcertés qu'instruits.

Je pense néanmoins, en alléguant la règle que j'ai énoncée, de façon peut-être compromettante, dans le premier paragraphe de ce compte rendu, que Robert Hébert parvient à gagner le pari qu'engage sa passion de la négativité (le dernier paragraphe du livre, p. 189) et que sa détermination à creuser une galerie sous la banalité des usages du langage quotidien et du discours philosophique parvient à produire un ébranlement dont je tenterai d'apprécier pour mon compte la profondeur. Il est sûr qu'en s'autorisant à comprendre d'avoir pensé en certains endroits de façon analogue, on s'expose de façon voyante au reproche de travestir. Je crois toutefois qu'à tout prendre cette stratégie est préférable au pacifique résumé, qui, de ne rien risquer, contrevient de façon radicale à un livre où tout n'est qu'inquiétude.

1. Quelques suggestions, d'abord, destinées à ceux qui sont déterminés par l'impératif de comprendre vite et par celui d'agir.

1.1 Pour ceux qui sont pressés de comprendre ou, à tout le moins, d'atténuer la singularité de ce texte, je proposerai une règle de lecture. Robert Hébert emploie dans son livre trois procédés d'exposition. Ces procédés, qui recoupent à la fois les grandes articulations d'un langage et les figures privilégiées du locuteur, posent des problèmes de décodage dont le degré de complexité est très différent. Le premier procédé, qui correspond à l'articulation paradigmatique de la langue et dont se sert l'enseignant pour produire des représentations synoptiques qui exhibent son travail, consiste dans la production de tableaux (le premier de ces tableaux se trouve à la page 27). Le second procédé, auquel le théoricien fait recours pour exprimer ses idées, tient dans l'enchaînement d'une suite de syntagmes qui constituent un discours argumentatif. Un troisième procédé, par lequel se marque une volonté d'insis- 
tance qui vise à affecter l'autre comme sujet, réside dans l'utilisation des caractères italiques. La règle de lecture que je propose est la suivante: d'observer dans la lecture d'un chapitre, quand il sera possible de le faire ${ }^{2}$, la séquence que $j$ 'ai moi-même suivie dans l'énumération des procédés d'exposition mis en cuvre par Robert Hébert. II faut donc prendre son point de départ dans l'analyse des tableaux et transformer celle-ci en un point d'appui pour progresser à travers le discours vers les plus énigmatiques des formules en italiques. Le tableau fonctionne souvent dans un texte à la manière d'une conclusion synoptique; il est préférable, dans le cas de Mobiles $d u$ discours philosophique, de lui donner le statut d'une initiation à des procédures d'expression dont le décodage présente des difficultés autrement considérables.

1.2 À ceux qui sont pressés d'agir, je conseillerai, s'ils sont enseignants, d'examiner leur pratique à l'étalon des propositions avancées par Hébert dans cette section du premier chapitre, intitulée "Équivoque instrumentale " (par. 45-49, pp. 57-61). Le marchandage des méthodes et des concepts de la philosophie comme étant autant d'instruments - dont la forme la plus prometteuse est celle de la " grille" - que l'étudiant n'aurait qu'à appliquer pour produire des connaissances est l'une des pratiques pédagogiques les plus propres à générer de ruineux malentendus sur ce qui peut être attendu de la philosophie et de son enseignement. Ce serait déborder le texte de Robert Hébert que de tenter de dresser la liste de toutes les fraudes que permet l'équivoque instrumentale. Leur commune matrice est toutefois clairement aperçue : «... tout pouvoir instrumental, écrit Robert Hébert en se référant à ce pouvoir instrumental qui est prédiqué de la réflexion, est pouvoir de quelque chose : l'instrument n'est efficace que dans l'accomplissement de sa fonction - sa fonction étant déjà inscrite dans le style de sa fabrication " (par. 46, p. 58, nous soulignons). En paraphrasant le texte d'Hébert: l'étudiant que l'on prétend instrumenter conceptuellement est amené à se persuader qu'il existe pour les outils qu'on lui

2. Tous les sous-chapitres ne comportent pas des tableaux qui reprennent de façon synoptique le résultat des analyses qui y ont été poursuivies. 
fournit un mode d'emploi inscrit dans leur facture même, communicable sous la forme d'un livret d'instruction et dont l'application méticuleuse donnera naissance de façon mécanique à des artéfacts culturels qu'on s'échangera en leur conférant la valeur de connaissances. Pour l'essentiel : l'équivoque instrumentale est ce leurre par lequel le sujet philosophant s'aveugle au surcrô̂t de détermination qui doit informer son pouvoir de penser pour que celui-ci produise un savoir.

2. Cette dernière proposition, qu'il nous appartient d'expliciter davantage, peut toutefois nous servir provisoirement d'aiguillage vers ce qui m'apparaît constituer l'un des motifs centraux du livre de Robert Hébert. Si javais à identifier ce motif, je dirais qu'il est le référent de l'une des expressions théoriques frappées par Hébert, qui possède un nombre suffisant d'occurrences pour apparaitre comme un leitmotiv parfois obsédant dans le texte de Mobiles du discours philosophique. Cette expression, par laquelle Robert Hébert veut rendre compte de l'investissement privilégié de la force de réflexion, est celle d'involution gratifiante vers l'intelligible (par. 52, pp. $65-66)^{3}$. Je m'emploierai maintenant à discuter quelques éléments des travaux de Robert Hébert qui nous permettront de circonscrire de façon plus exacte le motif qui vient d'être invoqué.

2.1 Il est, dans les archives de la philosophie, des propositions qui s'affirment comme des évidences, le cogito cartésien étant sans doute la plus notoire de ces propositions. Il se trouve également dans la pratique disciplinaire de la philosophie des rituels dont l'accomplissement constitue une performance requise par des consignes tacites qui, en vertu de leur banalité, ne sont jamais soupçonnées. Par exemple : "si je réfléchis bien (assez ou assez longtemps), je viendrai à savoir ", ou encore, sous la forme canonique d'une inférence cartésienne, « je pense, donc je sais ".

Que ce soit dans l'histoire de la pensée philosophique ou dans la pratique concrète de cette discipline, on peut faire état d'un certain nombre d'infractions à ces consignes, qui laissent

3. Cetre expression apparaît, avec quelques variantes, dans de nombreux autres passages : par exemple, par. 56, p. 71 ; par. 83 , p. 102 ; par. 86, p. 105. 
entrevoir la part de la police de culture à leur mise en force et à leur reproduction sous la forme de truismes. Robert Hébert fait explicitement référence à cette marge de la pensée, où l'on ne réfléchit à rien de précis, où l'on cherche sans trouver et où enfin on réfléchit "pour rien " (par. 51, p. 65). Il affirme en outre que « du point de vue de (sa) sémantique sauvage, ces expériences préliminaires sont extrêmement importantes » (par. 51, p. 65. On pourrait évidemment allonger la liste des exemples déjà cités). Pour ce qui est de la tradition philosophique, Robert Hébert nous reporte à ces épisodes refoulés qu'ont été la sophistique et le scepticisme (dans l'avant-propos, p. 5) et nous pouvons inférer que cette histoire transgressive de la philosophie a également été partie prenante dans la genèse de Mobiles du discours philosophique.

2.2 Essayons maintenant d'être plus clair sur la nature de ces expériences et, de façon plus générale, sur la part du négatif dans le passage de la pensée-philosophie au discours. Il faudrait à mon sens s'efforcer d'établir une distinction entre l'expérience du manque, qui constitue l'épreuve d'un discours à venir et dont on feint de ne pas posséder l'assurance, et celle d'une vacance si départie qu'elle constitue l'échec du pensable. Le manque est cette figure tacticienne du négatif, qui sollicite l'affirmation et qui en est déjà, au vrai, prégnante. Ce que je désigne comme vacance, en jouant de façon préméditée de l'ambiguïté (vacancy/boliday), ne peut par contre faire l'objet d'aucune métamorphose positive ni d'aucune réintégration culturelle. Les seuls résultats de ce passage à vide sont eux-mêmes aussi banals que les consignes de productivité qu'il enfreint. Par exemple : conquérir, après avoir cheminé dans un labyrinthe familier, la banale certitude qu'à tout ce qui est vécu comme problème ne se trouve pas une solution (sur laquelle on butera, à la condition de travailler assez fort) et percevoir en même temps qu'il est inutile de redoubler les italiques par des encres bigarrées pour atténuer la dérision de ce constat.

Il me semble que c'est d'abord une résistance à être expulsé de toutes ces places vacantes que s'efforce d'occuper la compulsion culturelle à produire, qui constitue le socle des travaux de Robert Hébert, (« . . . expérience qui aboutit à 
une sorte de ralentissement stupéfiant : celle d'enfoncer une porte ouverte (...) pour après coup ne plus pouvoir réfléchir », dernier paragraphe, p. 189). À témoin cet article qu'il publia dans Culture et langage sous le titre significatif de «De l'intolérabilité : remarques sur le vocabulaire de l'échec $»{ }^{4}$

On serait bien avisé de le parcourir de nouveau avant d'entreprendre la lecture de Mobiles du discours pbilosophique.

3. Les remarques qui précèdent avancent une hypothèse sur la primauté (ou sur le caractère antécédent) d'un type d'expérience dans la genèse des préoccupations qui ont conduit Robert Hébert à écrire l'ouvrage publié sous le titre de Mobiles $d u$ discours philosophique. Dans le texte même du livre, cette expérience-limite, où la pensée s'éprouve comme étant neutre par rapport aux impératifs traditionnels de se produire comme savoir et comme salut, se voit conférer un statut théoriquement dérivé. L'ordre des concepts inverse fréquemment la chronique de l'expérience.

3.1 Les expériences que nous avons précédemment alléguées sont en effet libérées dans le texte de Mobiles du discours philosopbique à la suite d'une opération qui fait l'axe même de toutes ces portes que Robert Hébert fait tourner. Cette opération établit une disjonction entre "l'usage neutre de la réflexion et (. . .) sa valeur de production aléthique " (par. 50, p. 63). L'usage neutre de la réflexion - la pratique d'une pensée neutralisante - est conçu dans les termes d'une énergétique : le penser s'y manifeste comme une force indifférenciée, une volonté encore indéterminée à se réaliser comme savoir. Sa valeur de production aléthique doit, par contre, se penser dans les termes d'une logique - d'un ensemble de signes perceptibles dans lesquels une culture reconnait les marques instituées de l'intelligible.

3.2.1 Les effets de cette opération sont multiples. Elle permet d'abord d'intégrer les résultats les plus significatifs des analyses qui précèdent son effectuation. Ces résultats s'étaient d'abord exprimés sous la forme de ce que Hébert appelle deux

4. Robert HÉBert (1973), "De l'intolérabilité : remarques sur le vocabulaire de l'échec ", in Collab., Culture et langage, Montréal, $\mathrm{HMH}$ (Cahiers du Québec, collection Philosophie, 11), p. 53-62. 
énonciations anthropologiques, qui recueillent une double représentation de l'activité de réflexion (voir les par.. 35 à 49). La première de ces deux représentations culmine dans l'énoncé d'une loi : l'exercice de la réflexion doit être continûment productif. La seconde représentation, qui ne fait que prolonger la première, manifeste la prise d'une décision : celle de subordonner les produits divers de la raison à cette forme privilégiée de l'activité rationnelle que constitue la réflexion (par. 42, p. 54). Or cette représentation double, à la fois productiviste et hiérarchisante, (je reproduis la terminologie d'Hébert), de la réflexion se révélera par la suite comme n’étant qu'une construction dont seule une logique qui disjoint l'usage neutralisant de la réflexion de sa valeur de production aléthique peut rendre compte. Cet aboutissement était prévisible puisque le développement consacré par Hébert aux deux énonciations anthropologiques s'était ouvert par une discussion de la duplicité culturelle du vocable "réflexion ", qui réfere autant à une activité (la réflexion) qu'à son résultat (la production de réflexions ; voir par. 36, pp. 49-50).

3.2.2 Un second résultat - le plus important - de la dissociation effectuée entre la force de penser et le produit culturel de l'application de cette force tient dans la conquête d'une attitude critique radicale (par. 50, p. 63), qui autorise l'interrogation s'énonçant dans le titre du livre. Dès lors, en effet, que le lien postulé entre l'acte de réfléchir et son produit aléthique culturel n'est plus aperçu comme étant nécessaire, une place est faite à l'examen des motifs - des mobiles - qui infléchissent la force de penser vers une volonté de savoir (par. 54, p. 68).

3.3 Cette question n'admet pas, en son principe, de solution explicite: considérée avant son extériorisation dans des produits-signes, l'activité de penser n'offre pas ou peu de prise à la description (par. 51, p. 65), et peut-être est-elle même en son fond inconcevable. L'interrogation à laquelle nous sommes parvenu peut toutefois amener ce que j'appellerai une stratégie de transgression.

Qu'est-ce à dire? Ne se pourrait-il pas que ce qui est refusé à un calcul démonstratif, dont toutes les propositions 
font l'objet d'un contrôle efficient, fût suggéré efficacement par le moyen d'une écriture qui fait pièce de tous les dérapages de la bonne conscience philosophique pour en subvertir les évidences? Robert Hébert avait à cet égard annoncé, dès le second paragraphe de Mobiles du discours philosopbique, que ce qu'il se projetait de faire était de "suggérer, par tous les moyens possibles (souligné dans le texte), cette morpho-génèse globale du discours philosophique qui occupe une position si particulière sur la scène culturelle »(par. 2, p. 10).

Je tenterai dans la suite de ce compte rendu de faire un examen (partiel) des résultats de cette stratégie de transgression. Je rappelle à cette fin mon présupposé : plutôt que de considérer le livre de Robert Hébert comme le développement d'une argumentation linéaire, je le conçois comme une circonférence tracée à partir d'un centre qui m'apparaît constitué par le clivage établi entre la force de penser et ses produits culturels extériorisés. Ce qui dans le livre précède cette coupure, l'annonce, et ce qui la suit en explore les résultats.

4. J'énoncerai une première série de transgressions dans l'horizon d'une question : que découvre-t-on lorsque l'on tente d'élucider la nature de la force de réflexion, considérée indépendamment de son produit? Pour l'essentiel, je crois, on trouve trois choses.

(i) La première de ces choses est un réseau de métaphores optiques et, partant, spatiales. Les résultats de l'analyse de ces réseaux de métaphores sont présentés, sous une forme résumée, dans les tableaux de la p. 27 et de la p. 37. Je ne me propose pas de commenter pour ma part ces résultats. On a souvent produit, depuis que Nietzsche l'a mise en formules, des illustrations de la thèse que certains des concepts fondateurs de la philosophie n'étaient que des métaphores, dont la nature figurative avait été recouverte par l'oubli.

(ii) La découverte d'un processus de métaphorisation n'est toutefois plus de celles qui clôturent une recherche. La métaphore est censée figurer quelque chose. Quoi ? Dans ce cas-ci, à vrai dire, rien. La partie du premier chapitre qui suit l'analyse des métaphores articulant la notion de réflexion 
débute en effet par un constat de pauvreté : « La lexicalisation philosophique de concept de réflexion, telle que l'exposent les instruments de travail en philosophie (lexiques, dictionnaires, vocabulaires, encyclopédies), s'avère extrêmement pauvre " (par. 26, p. 40). Ce constat doit s'entendre à la lettre: les enseignements de l'histoire de la philosophie n'autorisent qu'une caractérisation minimale de concept de réflexion. Ce concept n'est posé par Robert Hébert, en suite à son examen du corpus des textes de la philosophie, que comme un opérateur polymorphe de discours (par. 27, p. 41). Cette dernière expression n'est en réalité qu'une reduplication annonciatrice du clivage entre la force du réfléchir (opérateur polymorphe) et son produit discursif (de discours). En outre, elle paraît donner raison à $\mathrm{Hegel}$, qui affirme, dans sa Phénoménologie de l'Esprit, que les explications qui utilisent la terminologie de la force ne peuvent produire que des tautologies. De la force indifférenciée à l'opérateur polymorphe (et vice-versa), il ne semble pas que nous progressions, sinon dans le jeu de miroirs terminologique. L'impasse est toutefois bien davantage celle du corpus que celle de son analyste.

(iii) En effet, le caractère inexplicite de discours de la philosophie sur la nature des opérations réflexives qui l'engendrent est moins le fruit d'une indolence que celui d'une censure. En levant cette censure, Robert Hébert amène au discours ce qu'elle réprimait et qui constitue la honte de la famille des interdictions de la philosophie : derrière les silences de l'épistémologie s'entend la rumeur du psychologisme (de tous les repoussoirs de la philosophie, le plus décrié). Et si la réflexion n'était qu' " une sublimation (. . .) de l'expérience de l'attention "? (par. 48, p. 60). Cette hypothèse est dûment qualifiée par Robert Hébert de réductrice et même de caricaturale (par. 91, p. 109). Elle ne nous en conduit pas moins à commencer de tirer les conséquences les plus significatives de la disjonction initiale entre la force indifférenciée de réflexion et ses produits-signes. Le concept d'attention renvoie en effet de façon immédiate (comme en témoignent nos idiomatismes de langage) à celui d'effort (par. 89 à 95, pp. 108-113). Or cette transformation de la force en effort n'est plus une figure impuissante de la dérivation des analogies. Elle 
nous permet d'abord, en effet, de rétablir le lien provisoirement rompu entre les opérations de la réflexion et ses produits aléthiques : s'il est possible de se représenter la force de penser comme neutre, il est beaucoup plus problématique de concevoir un effort sans lui assigner un objet. Elle produit en second lieu un important changement de mode dans la démarche qui se poursuit dans Mobiles du discours pbilosophique: l'analyse descriptive engagée par une question sur la nature de la force de réflexion est relayée par une recherche des impératifs qui déterminent cet effort pour savoir.

5. Les réponses qu'apporte Robert Hébert à cette nouvelle question - pourquoi s'efforce-t-on de se déterminer à savoir? - produisent une nouvelle série transgressive. Cette seconde série constitue cependant à certains égards autant une redécouverte des positions classiques de la philosophie, qui étaient tombées en disgrâce ou en désuétude, qu'une sortie hors des lieux communs des philosophies actuellement prévalentes.

(i) Pourquoi donc le sujet de la philosophie s'efforce-t-il de produire un savoir? Un premier type de réponse s'énonce comme un thème récurrent dans le livre d'Hébert et allègue des impératifs d'ordre étbique (par ex. au par. 48, pp. 59-60 et à la fin, par. 139, p. 166). Il est difficile de donner à ces impératifs un autre contenu que celui de l'injonction, aussi vide qu'elle est péremptoire, de produire toujours plus de spéculations et d'expertises, que les multiples dispositifs de reproduction culturelle officialisent comme savoir. Cette difficulté, comme certaines des précédentes, ne ressortit cependant en rien aux lacunes de l'analyse; sa source se trouve dans le laconisme même des lieux communs d'une éthique de la productivité, qui requiert des chercheurs qu'ils se sacrifient à des écritures, dont ils seraient embarrassés de montrer la nécessité ou même la justification. Il est important de rappeler, par-delà la cassure établie par le positivisme entre l'épistémologie et l'éthique, que l'entreprise philosophique sollicite constamment, quand elle se publie comme un savoir, la caution d'une éthique (sentimentale).

(ii) Un retournement analogue à celui qui nous a fait découvrir sous le mutisme de l'épistémologie la loquacité d'une 
psychologie nous amènera maintenant à reconnaître en deçà de l'exhortation morale une volonté de jouir (par. 144, p. 170). Cette volonté est déterminée par le double rapport que le sujet philosophant entretient avec, d'une part, ce que Robert Hébert désigne sous l'appellation «la vie » et avec, d'autre part, la culture. Le désir du sujet de s'octroyer une jouissance qui contraste avec la frustration de sa vie quotidienne (par. 146, p. 172) l'engage d'abord à conférer à l'épreuve de son penser la valeur d'un affect (par. 160, p. 186). Ce passage au plaisir de soi pensant conduit Robert Hébert à définir la réflexion comme une « assomption élocutoire de la vie » (par. 139, p. 165). À la direction du mouvement près, ce thème se marque de façon insistante par la récurrence de l'expression « involution gratifiante vers l'intelligible », qui, dans Mobiles $d u$ discours philosophique, qualifie également l'effort de penser. Il faut souligner qu'en développant ce thème, Robert Hébert ranime l'antique tropisme sotériologique de la philosophie: toutes les métaphysiques du savoir s'achèvent par la position d'un « genre de connaissance » où le sujet éprouve sa pensée comme un affect porteur de salut.

(iii) Certaines formules utilisées par Robert Hébert laissent entendre que le désir du sujet de vivre l'exercice de sa réflexion comme une existence de substitution où il reçoit la prime de son intolérance envers les servitudes de la vie quotidienne est engendré par une illusion nourrie de l'extérieur. Cette croyance que la réflexion puisse compléter l'existence est en effet d'abord qualifiée de mythe (par. 74, p. 89). D'une autre façon, la thématique de la jouissance du penser est introduite dans le cadre de l'énoncé d'un postulat de l'expérience philosophique selon lequel "l'existence est conçue comme la consécration d'un corps individuel aux représentations, aux valeurs et aux fantasmes $d u$ corps culturel »(par. 143, p. 169, souligné dans le texte).

Ce corps culturel, défini comme complexe institutionnel et comme cumul d'archives (par. 144, p. 170), paraît en dernier lieu posséder une certaine extériorité par rapport au sujet pensant qui lui est asservi. Le postulat précité perd en effet une grande partie de sa signification si le corps culturel n'est investi d'aucune extériorité par rapport au corps individuel : 
s'y consacrer n'impliquerait alors rien d'autre que la plus conséquente et la plus austère des fidélités à soi-même.

6. Il est toutefois un passage important de Mobiles $d u$ discours philosophique qui se concilie mal avec les propositions (extrincésistes) précédentes. Je le citerai au long, car il condense ce qui, pour moi, fait dans une certaine mesure problème dans ce livre. Dans le paragraphe 67, Robert Hébert écrit :

"Le complexe institutionnel ne se développe pas à l'extérieur du discours philosophique; il n'en est pas un épiphénomène accidentel et par définition non philosophable. Au contraire, il croît de l'intérieur du penser philosophique si bien que le discours de ce dernier est l'effet de tout ce qui concrétise le complexe institutionnel. »

(P. 82, souligné dans le texte).

À y regarder d'un peu près, ce texte énonce une contradiction: l'engendrement du complexe institutionnel à partir de l'intérieur du penser philosophique implique l'antériorité logique du penser sur l'institution à laquelle il donne naissance ; l'affirmation que ce même penser est lui-même un effet du complexe institutionnel entraîne l'implication inverse de l'antériorité de l'institution sur le penser. Devant cette difficulté, le commentateur doit faire son choix entre deux options. II peut en première part la résoudre en espaçant selon une séquence temporelle deux truismes : (i) que l'institution doit d'abord se résoudre à épingler des pensées originales (antériorité logique du penser) et (ii) que les pensées ainsi institutionnalisées influent par choc en retour sur le processus de production de la pensée solitaire (on ne pense jamais qu'à l'intérieur d'une culture - position de l'antériorité historique de l'institution). L'exégète peut cependant choisir de subsumer le problème sous l'un des paradigmes des positions de crête auxquelles parvient une philosophie conséquente: l'immanence, qui tient autant à marquer la différence entre ce qui engendre et ce qui est engendré, qu'à nier que ces termes soient extérieurs l'un à l'autre. En clair, je l'espère : le penser s'engendrerait à partir de sa propre manifestation réifiée, à savoir le complexe institutionnel. Entre ces deux options, je choisis la seconde, qui introduit la tension plutôt que la 
banalité dans le livre de Robert Hébert et qui souligne également la part congrue qui y est faite à l'altérité véritable. De l'immanentisme au solipsisme, il n'y a pas si loin, et ce dernier terme désigne l'ornière familière à la pensée réflexive. Je ne suis pas assuré que Robert Hébert, fasciné par sa matière, ait complètement évité d'y glisser.

On ne peut en effet manquer d'être frappé de ce que Mobiles $d u$ discours philosophique constitue une sorte de monadologie du corps philosophant, dont toutes les portes et les fenêtres sur ce qui n'est pas philosophie semblent avoir été délibérément refermées.

(i) C'est en effet une règle méthodologique avérée de la sémantique, qui décrète que l'analyse tire une grande partie de sa pertinence d'approfondir les relations qui s'établissent entre des termes polaires. En dépit de sa réelle pauvreté, quand on la considère en elle-même, la théorie philosophique de la méthode réflexive aurait pu trouver un prolongement imprévu si Robert Hébert avait conduit ses analyses dans le profil de la différence qui affronte une philosophie réflexive à une science expérimentale. L'idée d'une science réflexive répugne au premier abord à nos préconceptions (le candidat le plus immédiat à ce statut contestable, la psychanalyse, se révèle à l'examen bien davantage comme la pratique d'un sujet écarté de soi par ses symboles qu'une mystifiante introspection). En explorant les raisons de cette répugnance, peut-être mal fondée, on aurait peut-être fini par buter sur l'inassimilable reste de la méthode réflexive en philosophie.

(ii) D'une façon analogue, le rapport à l'art (allégué de façon allusive dans l'avant-propos, p. 5) aurait fait surgir des différences justiciables d'une explication. Toute sublimation dans l'ordre du discours n'a pas pour fin, ni pour résultat, de superposer une intelligibilité supportable à l'intolérable de la vie (voir à cet égard la conclusion du livre, p. 191). À témoin, l'antisotériologie de Kafka, de Céline ou de Beckett, pour ne mentionner que les pratiques d'écriture (en peinture : Edvard Munch). Faire valoir l'argument que ces écrivains "se sont sauvés par l'écriture " équivaut à se fracasser sur l'écueil des poncifs les plus éculés de l'esthétique bourgeoise. 
(iii) Il est une dernière relation qui, si elle avait été considérée dans toute son étrangeté, aurait pu amener Robert Hébert à expliciter davantage certains de ses énoncés. C'est le rapport à autrui - l'intervention de l'autre sur soi. La genèse de la représentation productiviste de l'exercice de la réflexion reçoit un éclairage bien différent selon qu'on la conçoit comme la marque de l'intérêt de soi ou comme celle du souhait de l'autre. Il n'y a, à vrai dire, rien qui soit culturellement monstrueux dans la dérive improductive d'un penser pour autant qu'on s'abstienne de lui requérir un auditoire. C'est contraindre l'autre à témoigner de la gratuité satisfaite de cet exercice qui est proprement impensable. Non pas en vertu d'une morale douteuse du respect d'autrui, mais en raison d'une prédiction maintes fois avérée : l'invitation qui se dit dans un "Voulez-vous jouer avec moi " bute le plus souvent sur le "Seulement si j'y prends mon plaisir » d'autrui. C'est, dans de nombreux exemples, de cette contrainte de faire jouir l'autre que naît l'impératif de produire des énoncés qui intéressent.

7. Les remarques qui précèdent ne doivent pas s'entendre comme autant de réserves faites à une démarche, dont j'avoue qu'elle me passionne. Une entreprise qui tourne court est parfois justiciable d'une critique; quand elle parvient à atteindre son but, elle engage un prolongement.

Il est possible, à cet égard, qu'une réponse à des questions telles que "Pourquoi pensons-nous? " et "Pourquoi disonsnous notre pensée sur le mode du savoir ? » parvienne à s'énoncer dans le cadre d'un paradigme sotériologique dont l'acteur principal serait un sujet monastique qui recopie la Bible sans sortir de sa cellule, en possédant l'assurance de la sollicitude de son Dieu. Il me semble toutefois que la genèse du complexe institutionnel ne peut s'expliquer sans que l'on fasse intervenir le corrélat de l'intérêt d'enseigner et qui est l'obsession d'apprendre. (Cet intérêt et cette obsession, est-il besoin de le préciser, ne doivent pas s'interpréter d'une façon psychologique. Ce qu'ils engendrent, comme ce qui les détermine, est un corps institué.) La volonté de produire un savoir est le plus souvent infléchie par la requête de l'autre de 
s'approprier une vraisemblance. Le corps culturel n'est plus le corps gratifiant d'un Dieu, mais le corps complice et dévorant de l'Autre. Révéler les motifs de cette complicité inquiète, où l'intimidation prend parfois le relais de la complaisance, est une entreprise où Robert Hébert continuerait peut-être à trouver du plaisir.

Université de Montréal 